\title{
MODELING OF THE RADIATION INDUCED ELECTROMAGNETIC FIELD IN FINELY-DISPERSE MEDIA
}

\author{
M.E. ZHUKOVKIY ${ }^{1 *}$, V.A. EGOROVA ${ }^{1}$ \\ ${ }^{1}$ Keldysh Institute of Applied Mathematics RAS, Miusskaya sq. 4, Moscow, Russia \\ *Corresponding author. E-mail: usermath@mail.ru
}

\section{DOI: 10.20948/mathmontis-2021-52-6}

Summary. Algorithms for supercomputer modeling of the radiation electromagnetic field in heterogeneous materials of a complex finely-dispersed structure are constructed. A geometric model of a heterogeneous medium is created using Stilinger-Lubachevsky algorithms for multimodal structures. The model includes a system of detectors for statistical evaluation of functionals on the space of solutions of the photon-electron cascade transport equations. Algorithms for the three-dimensional approximation of the results of modeling the radiation transport in a fine-dispersed medium to an electrodynamic difference grid are developed. The approximation methods based on the technology of neural networks. The method of numerical solution of the complete system of Maxwell's equations for calculating the electromagnetic field in a fine-dispersed medium is worked out. The results of demonstration calculations of the electromagnetic field are presented. The results of the calculations show that the spatial distribution of the radiation electromagnetic field has a sharply inhomogeneous structure caused by the presence of boundaries of materials with different radiation properties.

\section{INTRODUCTION}

The investigations of radiation-induced (electromagnetic radiation, laser radiation, penetrating radiation) effects in media of complex geometrical structure are actual for a lot of applications: interaction EMP with objects [1], plasma generation and relaxation [2], ionizing radiation interaction with matter [3-5] and many others. Mathematical modeling is an effective method to such investigation [6-8].

The technique of a detailed supercomputer simulation of the processes of radiation-induced electrodynamic effects using ultra-high-performance computational techniques and modern parallelization technologies (MPI, OpenMP, CUDA) is presented in this paper.

The problems of mathematical modeling of radiation-induced charge and current effects in environments of complex geometric structure are considered in [9, 10]. Algorithms of supercomputer modeling of the formation of charge and current fields in heterogeneous polydisperse materials with direct resolution of their microstructure are described. The results of demonstration calculations of the parameters of charge and current fields are presented. The spatial distribution of radiation-induced charges has a sharply inhomogeneous structure due to the presence of boundaries of materials with strongly different radiation properties. Charge separation occurs near the boundary surfaces and can lead to the generation of a strong electric field that can disrupt the functional properties of a heterogeneous material with a finely dispersed structure.

Mathematical modeling of the radiation electromagnetic field includes the following tasks:

2010 Mathematics Subject Classification: 97M50, 97N50, 93A30.

Key words and Phrases: finely dispersed medium, radiation induced EMF, Monte Carlo simulation. 
- construction of a geometric model of a heterogeneous fine-dispersed medium, which includes a detector system for calculating the required values (energy deposit, electric current density) when modeling the interaction of radiation with matter;

- statistical modeling of radiation transport in a fine-dispersed medium with direct resolution of its microstructure;

- 3D approximation of the results of calculations of energy deposit and currents from the detector system used in solving the radiation transport problem to a spatial difference electrodynamic grid designed for numerical solution of the electrodynamics problem;

- numerical solution of the initial boundary value problem for the complete system of Maxwell equations.

The paper presents the results of modeling radiation-induced electromagnetic fields (EMF) in a fragment of a closed-cell structure, which consists of a binder and finely dispersed dielectric inclusions.

\section{GEOMETRIC MODEL OF A POLYDISPERSE MEDIUM FRAGMENT}

Let us consider a fragment of a fine-dispersed medium of a closed-cellular structure consisting of a binder and eight inclusions (Fig. 1). The binder material is polybutadiene (C4H6, density $\rho=0.95 \mathrm{~g} / \mathrm{cm}^{3}$ ), the inclusion material is ammonium perchlorate (NH4ClO4, $\left.\rho=1.95 \mathrm{~g} / \mathrm{cm}^{3}\right)$. The size of the fragment is shown in Fig. 1.

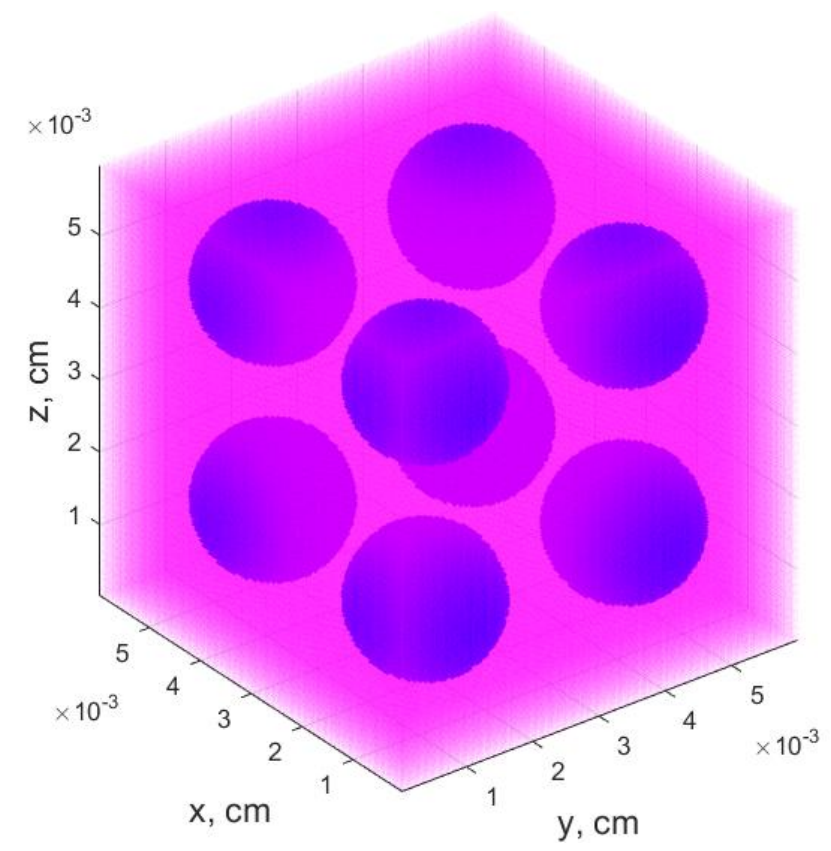

Figure 1: A fragment of a heterogeneous dispersed material

The geometric model of the medium includes a detector system for statistical evaluation of the required physical quantities. The detector (recording) system consists of a given number of spherical detectors of the same radius. The detectors should be isolated from each other (they should not intersect) and should not cross the boundaries of inclusions. 
The Stillinger-Lubachevsky algorithm and its modifications are used to construct a detector system of a polydisperse medium [11-13]. An example of the constructed detector system for the fragment under consideration is shown in Fig. 2.

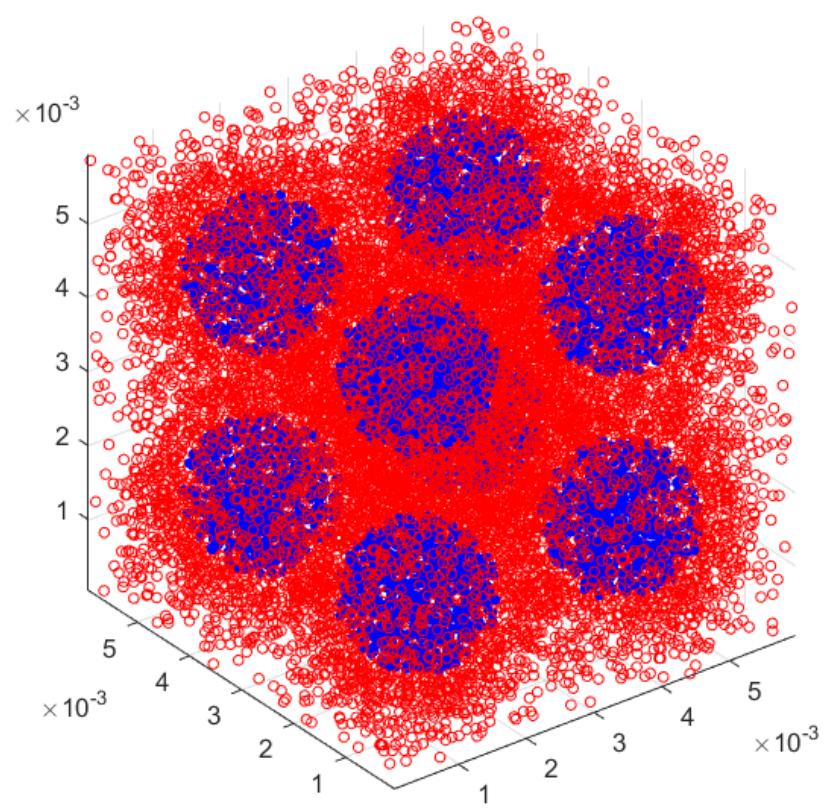

Figure 2: The detector system. Red color indicates detectors in the binder, blue - in inclusions

\section{MODELING OF RADIATION TRANSPORT IN A FINE-DISPERSED MEDIUM}

The processes of interaction of radiation with matter have a cascade character. The algorithms of statistical modeling of such processes are considered in detail in the works [14, 15]. These papers describe effective statistical algorithms for mathematical modeling of cascade radiation transport processes using hybrid computing technology. The algorithms are built considering the peculiarities of performing calculations on heterogeneous supercomputers using graphics accelerators as arithmetic calculators [14].

A modification of the processing scheme of the "tree" describing the cascade of particles by generations is proposed and implemented. The modification is developed on the basis of the use of stacks for temporary storage of information about the particles being born. The algorithm for filling these stacks takes into account a priori information about the relative path length of particles of various varieties.

The created method is optimized in terms of minimizing the amount of information required for processing the cascade tree. The approach to the organization of calculations in modeling the emergence and development of a photon-electron cascade is improved to achieve maximum GPU performance and increase the efficiency of simultaneous CPU/GPU loading. The algorithm for registering unlikely events is optimized in order to increase the information value of photon trajectories.

Figure 3 shows an example of the result of calculating the energy deposit in the considered dispersed fragment in the case when the object is irradiated by a photon flux with an energy of $20 \mathrm{keV}$. The direction of propagation of a flat flow is along the $\mathrm{Z}$ axis. 


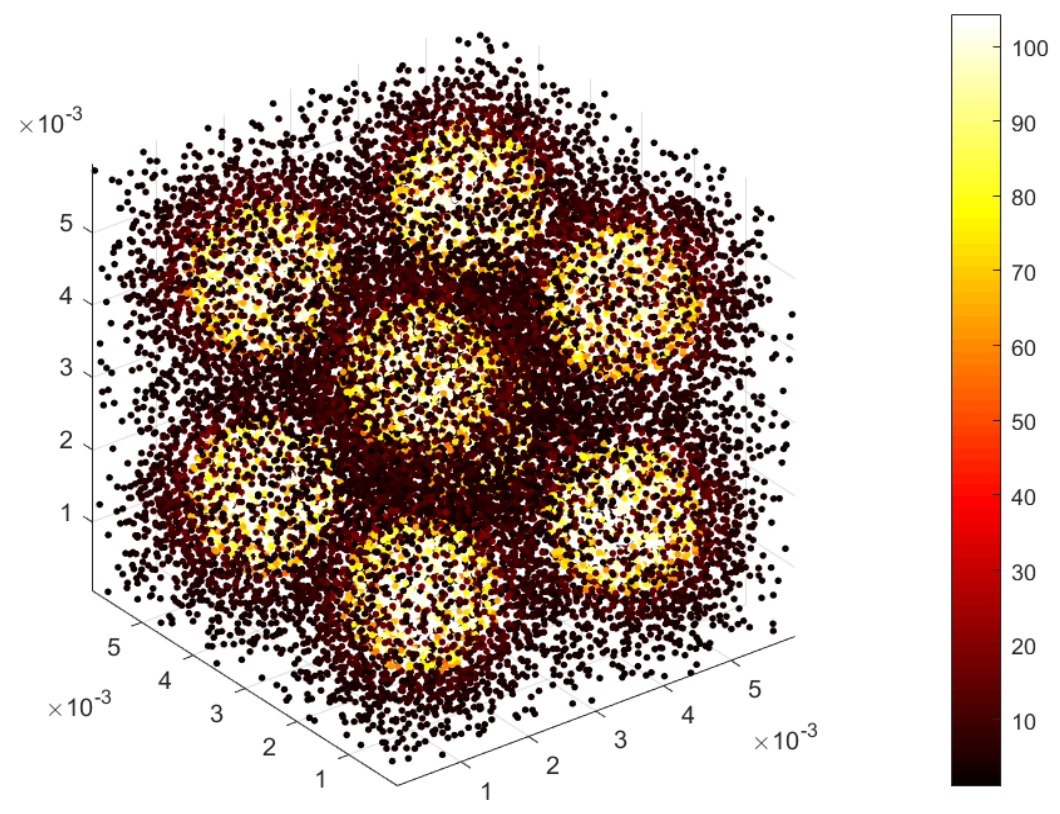

Figure 3: Results of calculation of the energy deposit density $\left(\mathrm{keV} / \mathrm{cm}^{3}\right)$

\section{APPROXIMATION OF THE RESULTS OF MODELING THE RADIATION TRANSPORT TO AN ELECTRODYNAMIC GRID}

Modeling of the radiation electromagnetic field requires the joint use of different software tools to assess the influence of various interdependent factors on the functional properties of the materials under study.

The use of mathematical models and numerical methods for computer research of processes of various physical nature (interaction of radiation with matter, secondary electrodynamic effects) makes it necessary to use various geometric approximations to describe the object. In this regard, there is a problem of integrating "according to data" the results of a numerical study of various physical processes in various mathematical models.

The problem of adequate transfer of the results of statistical modeling of the radiation energy deposit and radiative electric currents from the detector system used in modeling the interaction of radiation with matter to a rectangular Cartesian electrodynamic difference grid is solved using various approximation methods based on the technology of machine learning [16], in particular, on the technology of neural networks [17].

A multilayer perceptron [17] was used to solve approximation problems in this paper.

The network topology (3-100-30-1) and the logistic function $f(x)=1 /\left(1+e^{-x}\right)$ of neuronal activation [17] were used to approximate the energy deposit density.

Figure 4 presents as an example the result of approximating the energy deposit density on an electrodynamic grid in the form of a surface in the plane $\mathrm{z}=0.0015 \mathrm{~cm}$. 


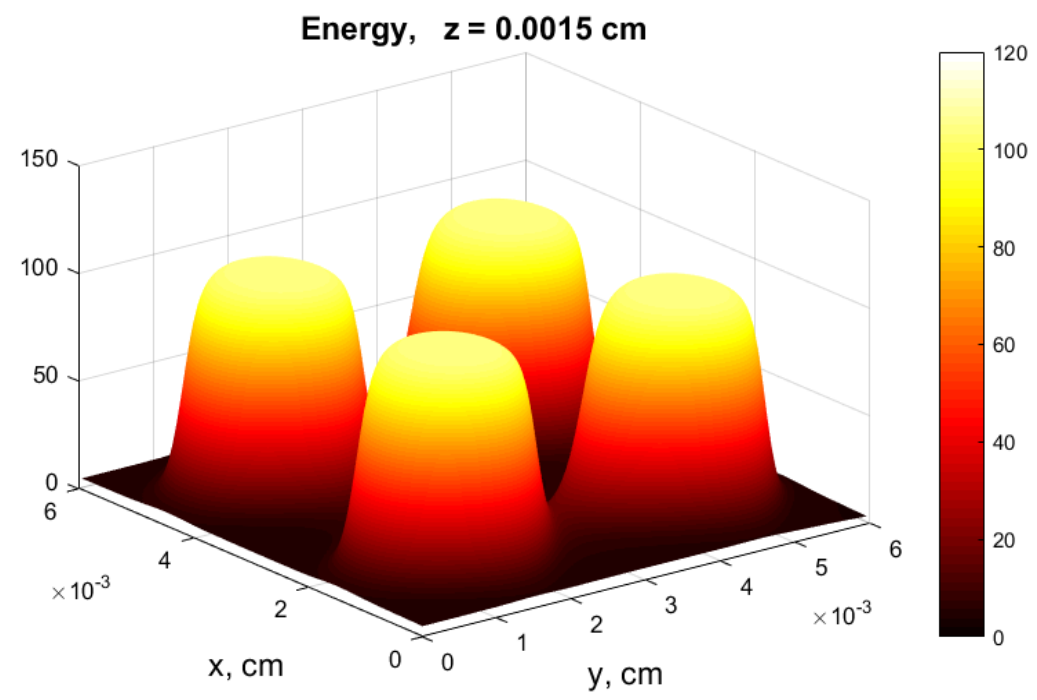

Figure 4: The energy deposit density in the plane $\mathrm{z}=0.0015 \mathrm{~cm}\left(\mathrm{keV} / \mathrm{cm}^{3}\right)$

The network topology (3-60-25-8-1) for approximating the current components is used.

Different activation functions are used for approximating the current components. Tangential function $(f(x)=\tanh (x))$ is applicated for the transverse current components $J_{x}$ and $J_{y}$ because the transverse components $J_{x}$ and $J_{y}$ are odd relative to the center of the fragment and logistic one $\left(f(x)=1 /\left(1+e^{-x}\right)\right)[4,17]$ is used for the longitudinal component $J_{z}$. The electrodynamics grid has size $100 \times 100 \times 100$.

As an example, the results of approximation of the transverse components of the electron flux density to the electrodynamic grid are presented in Fig. 5, 6. The results are depicted as surfaces on a slice $\mathrm{z}=0.0015 \mathrm{~cm}$.

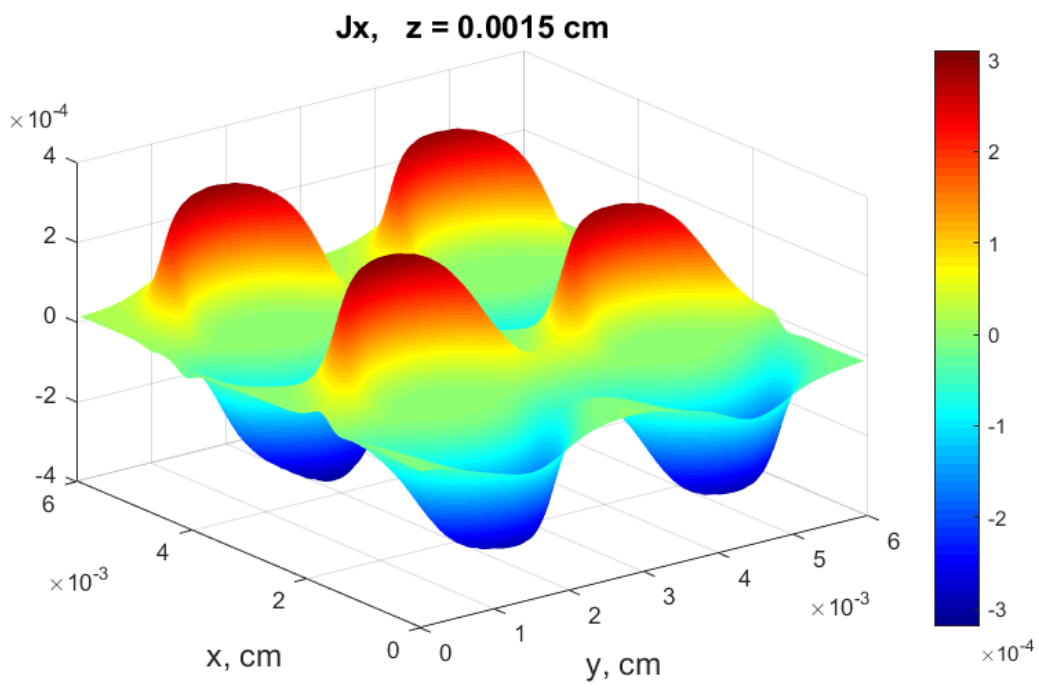

Figure 5: Function $J_{x}(x, y), \mathbf{z}=0.0015 \mathrm{~cm}, 1 / \mathrm{cm}^{3}$ 


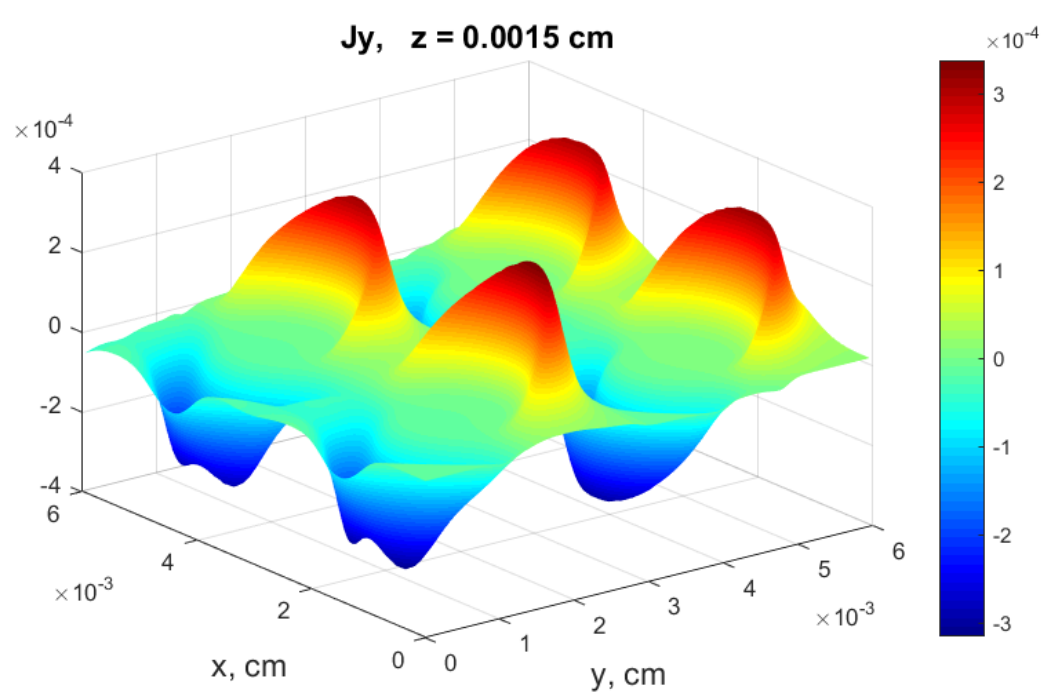

Figure 6: Function $J_{y}(x, y), \mathbf{z}=0.0015 \mathrm{~cm}, 1 / \mathrm{cm}^{3}$

A constant step of gradient descent with the BFGS optimization method (a quasiNewtonian optimization method with the Broyden-Fletcher-Goldfarb-Shanno scheme [18]) is chosen to solve the approximation problem.

\section{ELECTRODYNAMIC MODEL}

The EMF generation process is described by a complete system of nonstationary Maxwell equations:

$$
\begin{gathered}
\operatorname{rot} \mathbf{H}=\varepsilon \frac{1}{c} \frac{\partial \mathbf{E}}{\partial t}+\frac{4 \pi}{c}(\sigma \mathbf{E}+\mathbf{j}), \\
\operatorname{rot} \mathbf{E}=-\mu \frac{1}{c} \frac{\partial \mathbf{H}}{\partial t}, \\
\frac{\partial \rho}{\partial t}+\operatorname{div}(\sigma \mathbf{E}+\mathbf{j})=0, \\
\mathbf{j}=\varphi(t)(\delta(z), 0,0),\left.\mathbf{E}\right|_{t=0}=\left.\mathbf{H}\right|_{t=0}=\left.\rho\right|_{t=0}=0 .
\end{gathered}
$$

In (1):

$\rho=\rho(t, \mathbf{r})$ - electric charge density,

$\mathbf{j}=\mathbf{j}(t, \mathbf{r})$ - electric current density,

$\varepsilon=\varepsilon(\mathbf{r})-$ dielectric constant of material,

$\mu=\mu(\mathbf{r})-$ magnetic constant of material,

$\sigma=\sigma(\mathbf{r})-$ conductivity of materials.

Let's consider equations (1) in a bounded domain $\Omega=\left\{x, y, z: x \in\left[x_{\min }, x_{\max }\right], y \in\left[y_{\min }, y_{\max }\right], z \in\left[z_{\min }, z_{\max }\right]\right\} \quad$ with a boundary $\partial \Omega$. The 
condition $\iint_{\partial \Omega}\langle[\mathbf{E}, \mathbf{H}] \cdot \mathbf{n}\rangle d s . .0$, is considered fulfill at the boundary $\partial \Omega$ of the region $\Omega$ (n is a unit vector in the direction of the external normal to the surface $\partial \Omega$ ).

The equality of the tangential components of the electric and magnetic fields at the boundary is used as approximate boundary conditions.

$$
\begin{aligned}
& E^{y}=H^{z} \quad E^{z}=-H^{y} \text { at } x=x_{\max }, \quad E^{y}=-H^{z} \quad E^{z}=H^{y} \quad \text { at } x=x_{\min }, \\
& E^{z}=H^{x} \quad E^{x}=-H^{z} \text { at } y=y_{\max }, E^{z}=-H^{x} \quad E^{x}=H^{z} \text { at } y=y_{\min } \text {, } \\
& E^{x}=H^{y} \quad E^{y}=-H^{x} \quad \text { at } \quad z=z_{\max }, \quad E^{x}=-H^{y} \quad E^{y}=H^{x} \text { at } \quad z=z_{\min } \text {, } \\
& t \geq 0, \mathbf{r} \in \Omega .
\end{aligned}
$$

The conditions (2) ensure the coincidence of the directions of the Poynting vector and the external normal to the boundary, allowing the outflow of electromagnetic energy from the area and prohibiting the inflow. The boundary conditions (2) are called "radiation conditions".

\section{NUMERICAL ALGORITHM FOR SOLVING MAXWELL'S EQUATIONS}

The algorithm for the numerical solution of the initial boundary value problem for system (1) with the boundary conditions (2) is based on the difference scheme presented in $[19,20]$. It has proven itself well in solving a large number of different electrodynamic problems [20].

Let's consider Maxwell's equations (1) in the Cartesian coordinate system $\mathbf{r}=(x, y, z)$ :

$$
\begin{gathered}
\partial_{y} H^{z}-\partial_{z} H^{y}=\varepsilon \dot{E}^{x}+I^{x}, \\
\partial_{z} H^{x}-\partial_{x} H^{z}=\varepsilon \dot{E}^{y}+I^{y}, \\
\partial_{x} H^{y}-\partial_{y} H^{x}=\varepsilon \dot{E}^{z}+I^{z}, \\
\partial_{z} E^{y}-\partial_{y} E^{z}=\mu \dot{H}^{x}, \\
\partial_{x} E^{z}-\partial_{z} E^{x}=\mu \dot{H}^{y}, \\
\partial_{y} E^{x}-\partial_{x} E^{y}=\mu \dot{H}^{z},
\end{gathered}
$$

the symbols $\partial_{x}, \partial_{y}, \partial_{z}$ mean partial derivatives with respect to the corresponding coordinates, the dot above the function denotes its partial derivative with respect to the variable $\xi \equiv c t,\left\{\xi: \xi \in\left[0, \xi_{\max }\right]\right\}, \mathbf{I} \equiv(4 \pi / c)(\sigma \mathbf{E}+\mathbf{j})$.

The difference grid for the variable $x$ is as follows:

$x_{i+1}=x_{i}+\Delta_{i} ; \quad i=0, \ldots, N_{x}-1, \quad x_{0}=x_{\min }, \quad x_{N_{x}}=x_{\max } ;$

$x_{i+1 / 2}=\left(x_{i}+x_{i+1}\right) / 2 ; \quad i=0, \ldots, N_{x}-1, \quad x_{-1 / 2}=x_{0}, \quad x_{N_{x}+1 / 2}=x_{N_{x}}$;

$\delta_{i}=x_{i+1 / 2}-x_{i-1 / 2} ; \quad i=0, \ldots, N_{x}, \quad \delta_{0}=\Delta_{0} / 2, \quad \delta_{N_{x}}=\Delta_{N_{x}-1} / 2$.

For variables $(y, z)$, the difference grid is introduced in the same way.

We choose a grid so that the discontinuities of the coefficients of the equations are located on the surfaces $x=x_{i}, y=y_{j}$, and $z=z_{k}$. The coefficient values are set at grid points with fractional indexes. These points coincide with the centers of rectangular parallelepipeds 
formed by the intersection of the planes $x=x_{i}, x_{i+1}, y=y_{j}, y_{j+1}$, and $z=z_{k}, z_{k+1}$. All the coefficients of the system, current densities and components of the electromagnetic field are continuous inside these parallelepipeds.

The component of the electric field normal to the rupture surface $\varepsilon$ suffers a rupture when passing through this surface. The components of the electric field tangent to this surface are continuous in this case. Therefore the grid components of the electric field $E^{x}, E^{y}$ and $E^{z}$ are defined in the middle of the corresponding edges of these rectangular parallelepipeds.

The components of the magnetic field are placed in the centers of the faces of the parallelepipeds.

Finite-difference analogs of equations (3-8) are given in [20].

A nonuniform time grid is introduced to construct a difference approximation of Maxwell's equations in time on the interval $t \in\left[t_{\min } ; t_{\max }\right]$ :

$$
\begin{gathered}
t_{n+1}=t_{n}+\Delta t_{n} ; \quad n=0, \ldots, N_{t}-1, \quad t_{0}=t_{\min }, \quad t_{N_{t}}=t_{\max }, \\
t_{n+1 / 2}=\left(t_{n}+t_{n+1}\right) / 2 ; \quad n=0, \ldots, N_{t}-1, \\
\delta t_{n}=t_{n+1 / 2}-t_{n-1 / 2} ; \quad n=2, \ldots, N_{t}-1 .
\end{gathered}
$$

The components of the electric field $\left(E^{x}, E^{y}, E^{z}\right)$ are given at integer moments of time $t_{n}$. The components of the magnetic field $\left(H^{x}, H^{y}, H^{z}\right)$ and the electric current density $\left(j^{x}, j^{y}, j^{z}\right)$ are in half-integer moments of time $t_{n+1 / 2}$.

The time derivatives are approximated by explicit difference equations [20]:

$$
\begin{gathered}
\partial_{t} E^{x}=\frac{E_{i+1 / 2, j, k, n+1}^{x}-E_{i+1 / 2, j, k, n}^{x}}{\Delta t_{n}} ; \quad \partial_{t} H^{x}=\frac{H_{i, j+1 / 2, k+1 / 2, n+3 / 2}^{x}-H_{i, j+1 / 2, k+1 / 2, n+1 / 2}^{x}}{\delta t_{n+1}} ; \\
\partial_{t} E^{y}=\frac{E_{i, j+1 / 2, k, n+1}^{y}-E_{i, j+1 / 2, k, n}^{y}}{\Delta t} ; \quad \partial_{t} H^{y}=\frac{H_{i+1 / 2, j, k+1 / 2, n+3 / 2}^{y}-H_{i+1 / 2, j, k+1 / 2, n+1 / 2}^{y}}{\delta t_{n+1}} ; \\
\partial_{t} E^{z}=\frac{E_{i, j, k+1 / 2, n+1}^{z}-E_{i, j, k+1 / 2, n}^{z}}{\Delta t_{n}} ; \partial_{t} H^{z}=\frac{H_{i+1 / 2, j+1 / 2, k, n+3 / 2}^{z}-H_{i+1 / 2, j+1 / 2, k, n+1 / 2}^{z}}{\delta t_{n+1}} .
\end{gathered}
$$

The constructed numerical algorithm is implemented in the form of a software module focused on multiprocessor computing equipment [20] using MPI parallelization technology. 


\section{RESULTS OF MODELING OF THE RADIATION ELECTROMAGNETIC FIELD}

Some results of modeling the radiation electromagnetic field in a heterogeneous finedispersed medium are presented in this section.

The object of irradiation is a fragment of a binder (polybutadiene) and dielectric inclusions (ammonium perchlorate) shown in Fig. 1.

The power of the gamma radiation source is described by the function

$$
J=N_{0} f(t), f(t)=\frac{2}{t_{0}}\left(1-\frac{t}{t_{0}}\right), t_{0}=2 \cdot 10^{-8} c ; \quad \int_{0}^{t_{0}} f(\tau) d \tau=1 .
$$

$N_{0}$ is selected in such a way that the amplitude of the electric field is of the order of 1 CGSE.

The radiation conductivity of the materials was determined by the formulas [12]:

$\sigma=1.8 \cdot 10^{-7} \mathrm{D}$ for the binder and $\sigma=1.8 \cdot 10^{-6} \mathrm{D}$ for inclusions, $D$ is the radiation dose rate in $\mathrm{rad} / \mathrm{s}[21]$.

Two-dimensional distributions of the electric field amplitude in planes orthogonal to the coordinate axes are presented in Fig. 7-10 (colormap is "jet").

Values of fields are in CGSE.

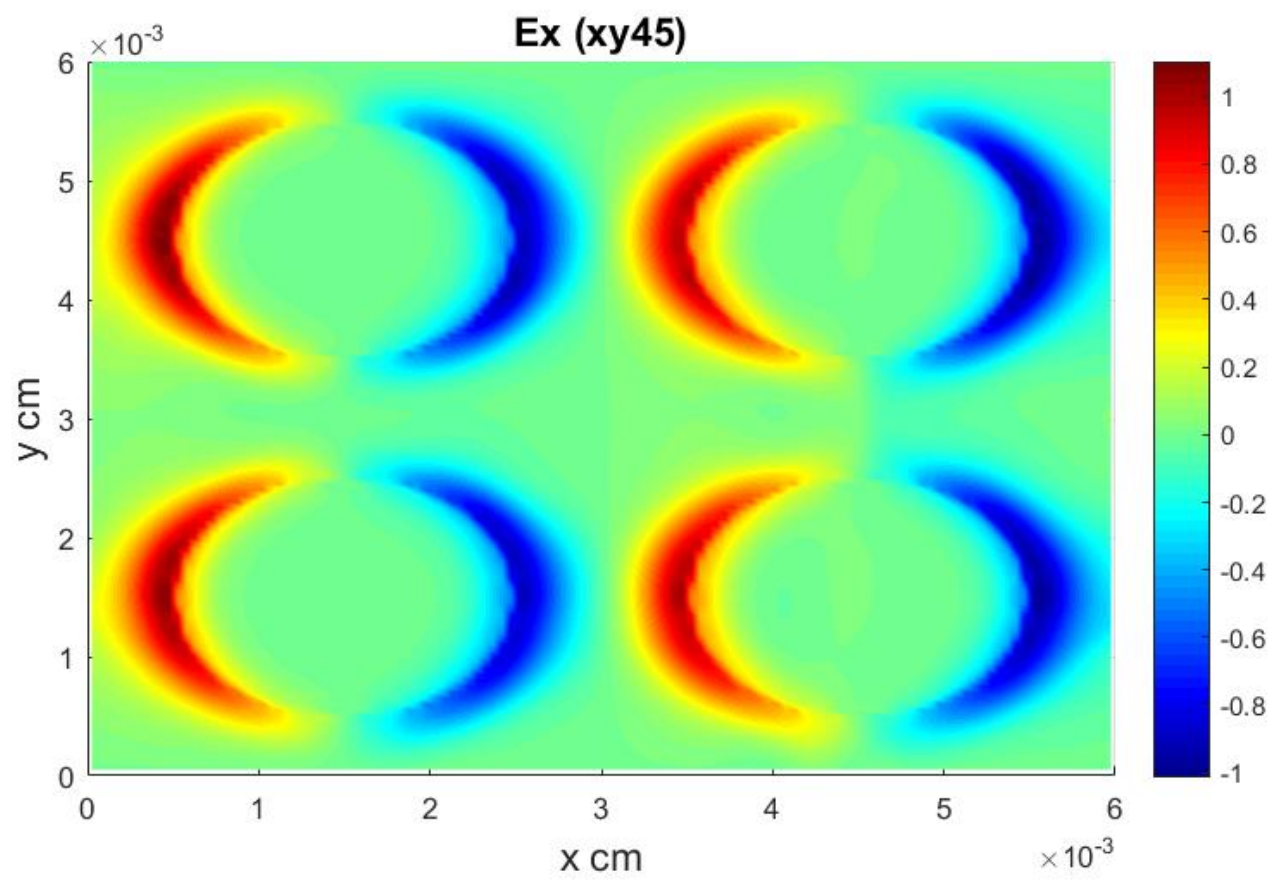

Figure 7: $E_{x}, z=0.0045 \mathrm{~cm}$ 


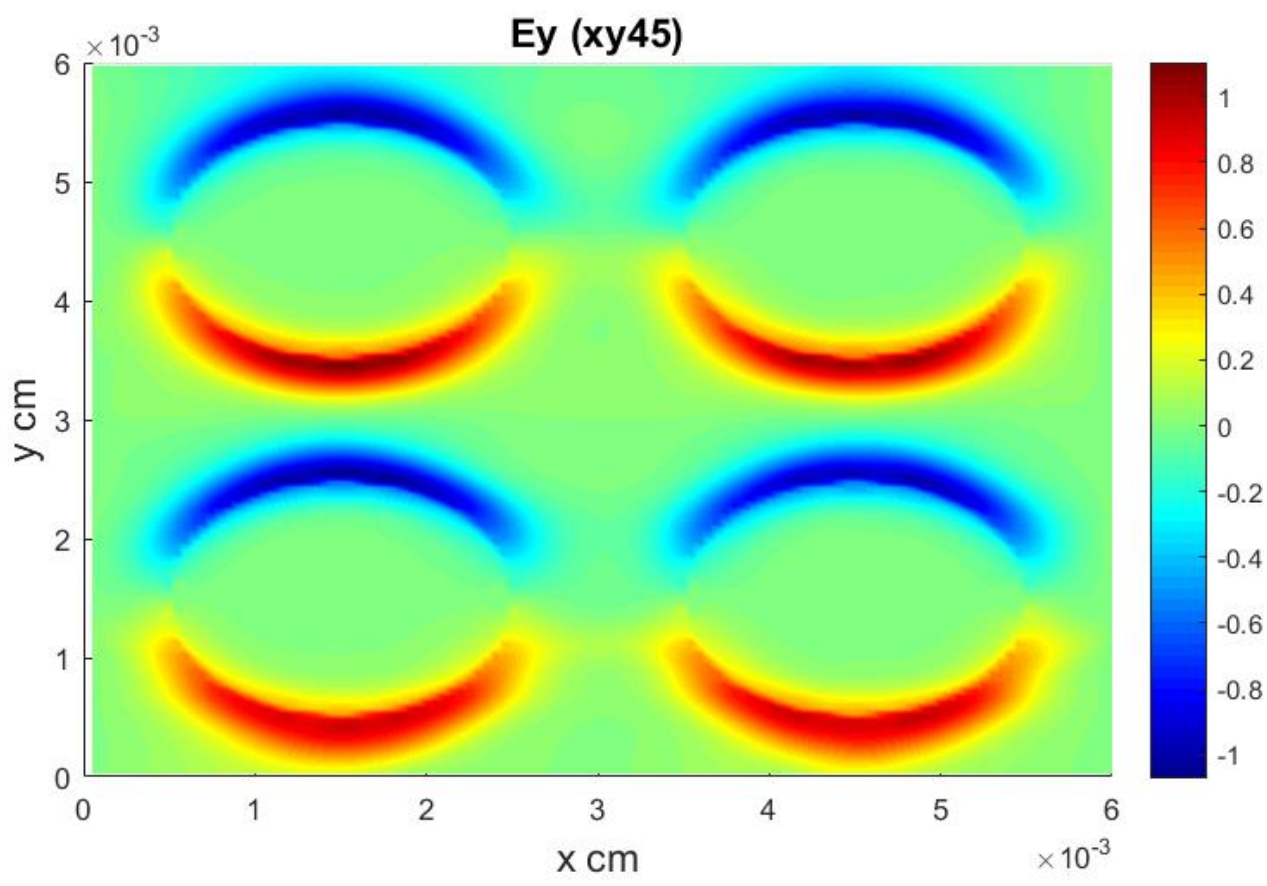

Figure 8: $E y, z=0.0045 \mathrm{~cm}$

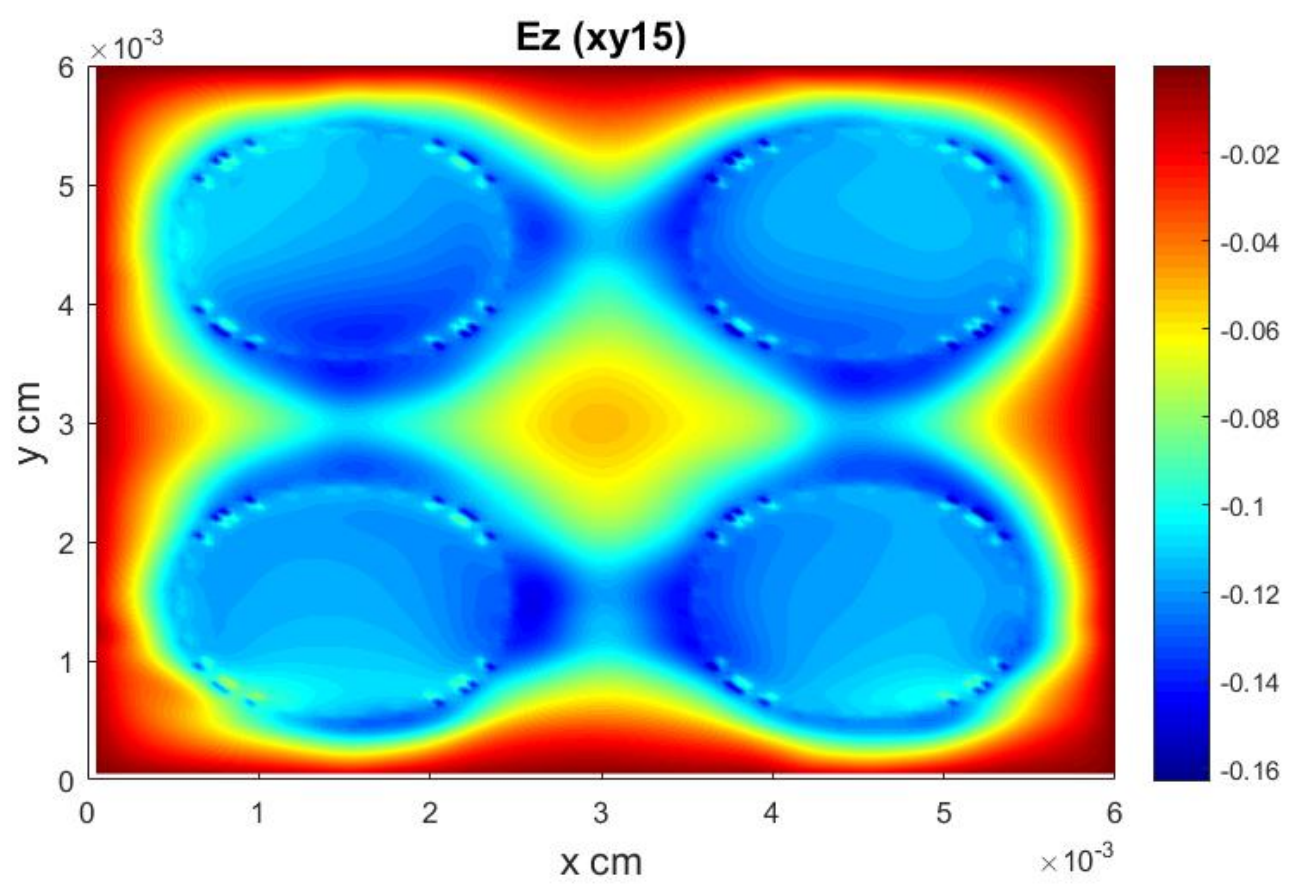

Figure 9: $E z, z=0.0015 \mathrm{~cm}$ 


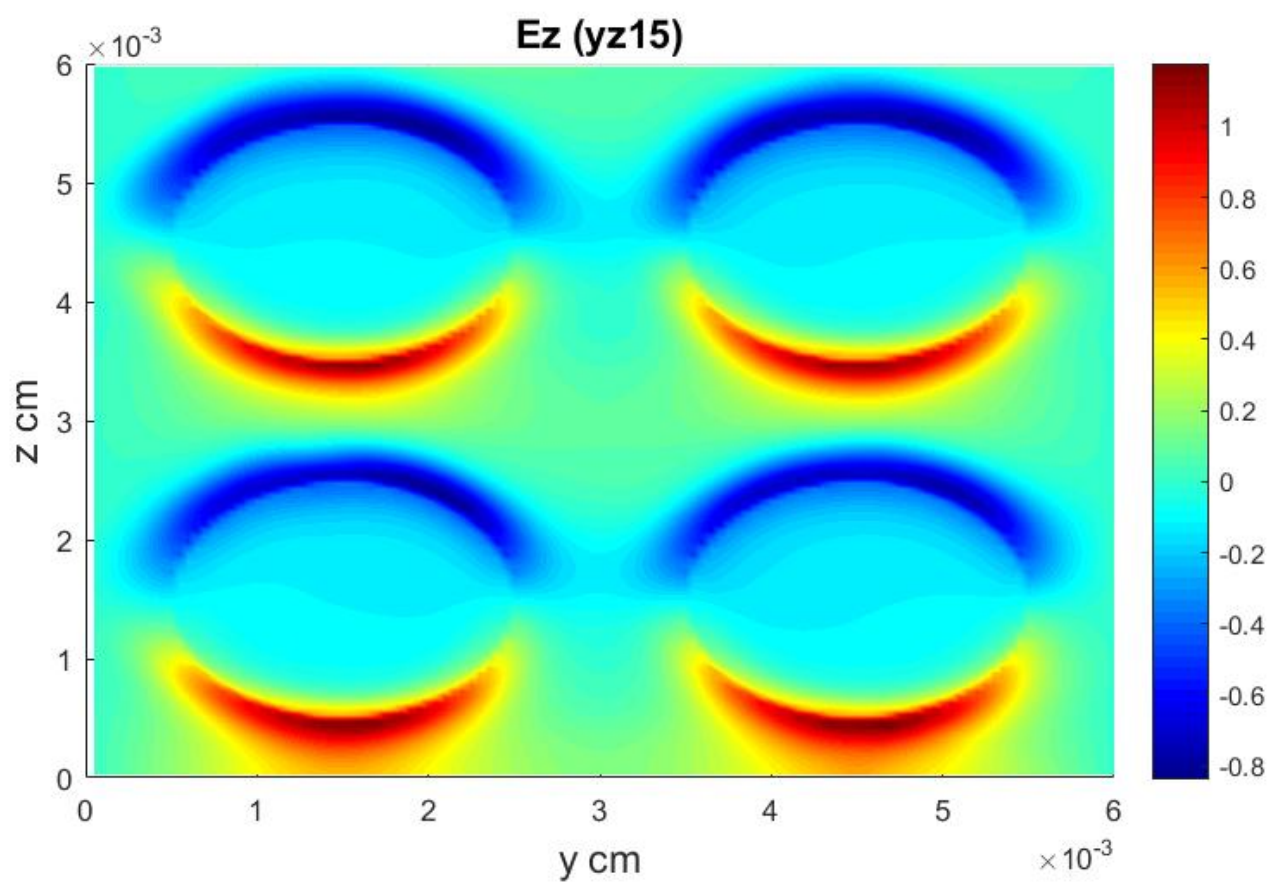

Figure 10: $E z, x=0.0015 \mathrm{~cm}$

Two-dimensional spatial distributions of the amplitudes of the transverse (relative to the direction of the photon flux) components of the magnetic field in the plane $\mathrm{z}=0.0045 \mathrm{~cm}$ are presented in Fig. 11, 12.

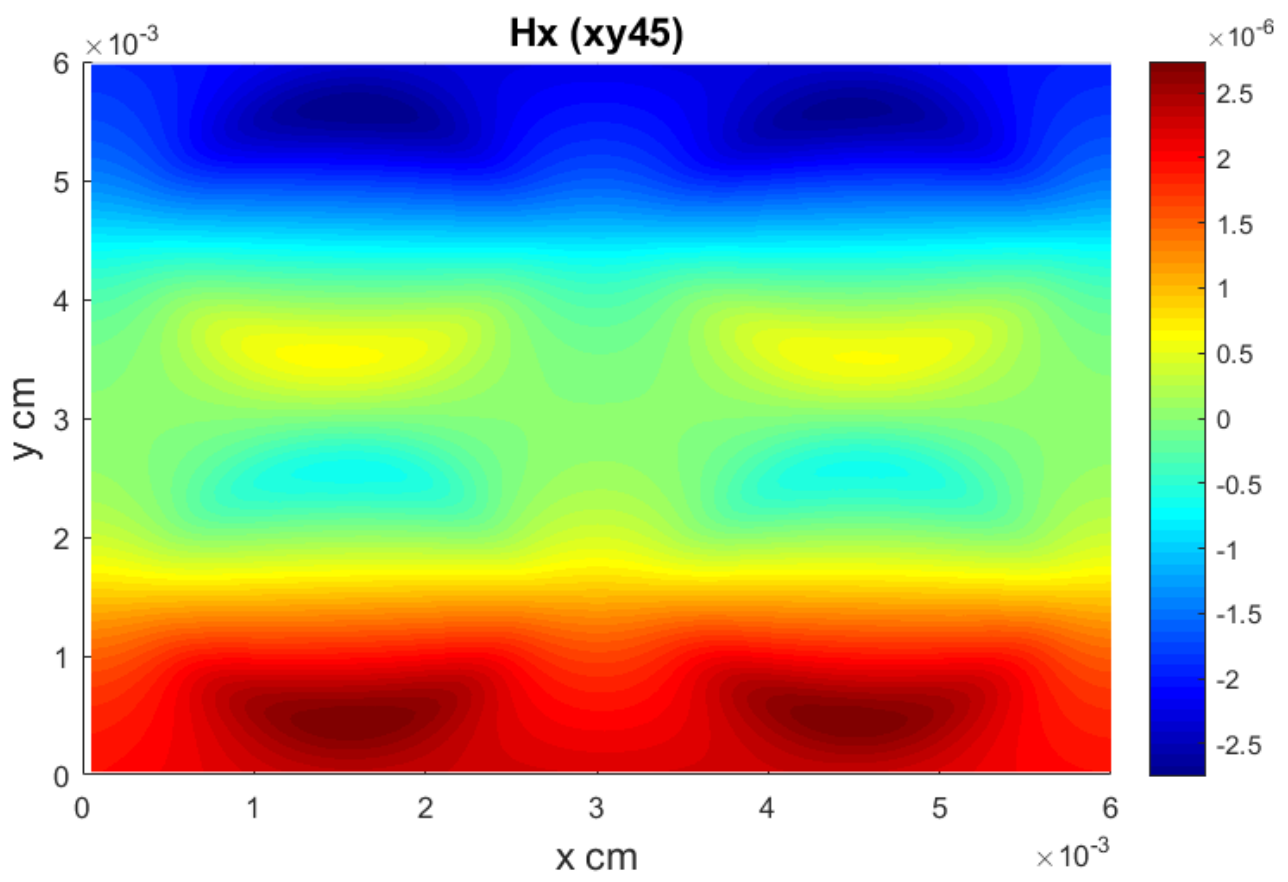

Figure 11: $H x, z=0.0045 \mathrm{~cm}$ 


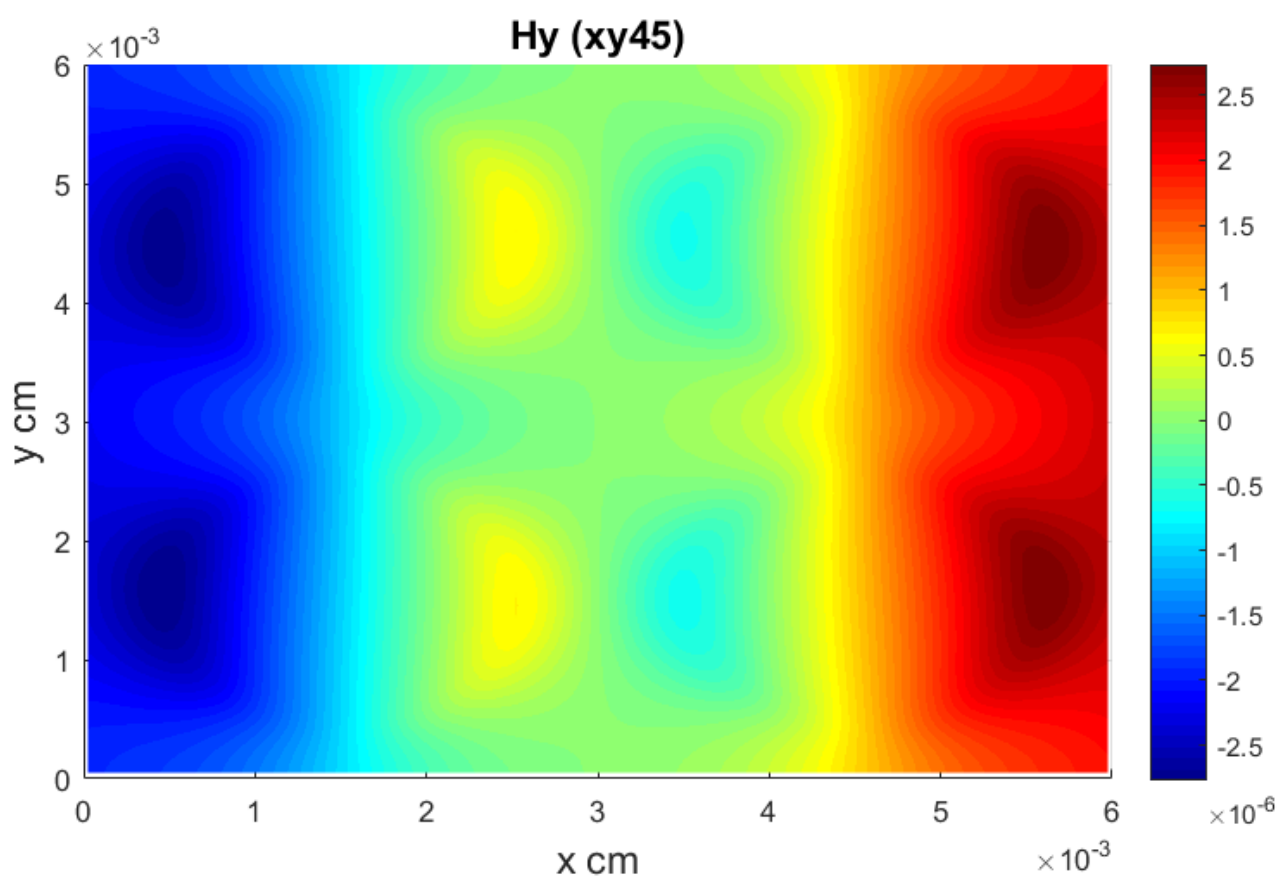

Figure 12: $H y, z=0.0045 \mathrm{~cm}$

The above illustrations show that when the fragment under study is irradiated, electric fields with a sharply inhomogeneous spatial structure are generated, and inhomogeneities occur near the boundar. The generated magnetic field is negligible compared to the electric one.

\section{CONCLUSIONS}

Algorithms for supercomputing the radiation electromagnetic field in heterogeneous finedispersed materials with direct consideration of their microstructure have been developed. The modeling includes a statistical evaluation of radiation energy deposit and electric currents, an approximation of the results from the detector system to the difference electrodynamic grid, and a numerical solution of the complete system of Maxwell's equations.

A geometric model of a heterogeneous fine-dispersed medium is constructed. The model includes the detecting system for statistical estimation of functionals on the space of solutions of the photon-electron cascade transport equations.

A mathematical three-dimensional simulation of the radiation EMF in a fragment of an object of a dispersed structure was carried out. The results of modeling show that electromagnetic fields with a sharply inhomogeneous spatial structure are formed and inhomogeneities of the fields occur near the boundary surfaces of the binder and inclusions.

The generation of EMF at the boundary of two media is due to the predominance of electron emission from the inclusion (a material with a large macroscopic cross-section of photons) into the binder (a material with a greater penetrating ability of electrons) over the emission in the opposite direction. 


\section{REFERENCES}

[1] R. Fisher, F. W. Smith and K. Cho, "EMP Coupling to Canonical Models Consisting of Dielectric and Metal Sections with Emphasis toward Simple Missile Models," IEEE Transactions on Nuclear Science, 28 (6), 4490-4494, (1981), doi: 10.1109/TNS.1981.4335752.

[2] V. I. Mazhukin, M. M. Demin and A. V. Shapranov, "High-speed Laser Ablation of Metal with Pico- and Subpicosecond Pulses", ASS, 302, 6-10 (2014).

[3] P. A. Childs, D. W. Dyke, "Analytic expressions for impact ionization rates and secondary particle energy distributions in semiconductors", Appl. Phys. Lett., 74, 2646 (1999); https://doi.org/10.1063/1.123925.

[4] M. E. Zhukovskiy, M. B. Markov, R. V. Uskov, "Modeling of radiation-induced electric current in the materials of finely dispersed structure", Math. Montis., 47, 65-74 (2020).

[5] A. V. Berezin, Yu. A. Volkov, M. B. Markov, I. A. Tarakanov, "The radiation-induced conductivity of silicon". Math. Montis., 33, 69-87 (2015).

[6] F.N. Voronin, K.K. Inozemtseva, M.B. Markov, "Electromagnetic and Thermomechanical Effect Produced by an Electronic Beam on a Solid Barrier", Math. Models Comput. Simul., 10, 407-417 (2018). Doi.org/10.1134/S2070048218040154.

[7] M.E. Zhukovskiy, R.V. Uskov, E.B. Savenkov, M. V. Alekseev, M. B. Markov, F. N. Voronin, "Model for the Radiation Transport in the Matter of Porous-Type Heterogeneous Materials", Math. Models Comput. Simul., 11, 415-425 (2019). https://doi.org/10.1134/S2070048219030177.

[8] V. A. Egorova, F. N. Voronin, M. E. Zhukovskiy, M. B. Markov, A. I. Potapenko, R. V. Uskov, D. S. Boykov, "Model of Radiation-Induced Thermomechanical Effects in Heterogeneous Finely Dispersed Materials", Math. Models Comput. Simul., 12, 729-739 (2020). https://doi.org/10.1134/S2070048220050063.

[9] M. E. Zhukovskiy, M. B. Markov, R. V. Uskov, "Modeling of radiation-induced electric current in the materials of finely dispersed structure", Math. Montis., 47, 65-74 (2020).

[10] . M.V. Alekseev, V.A. Egorova, M.E. Zhukovskiy, D.N. Sadovnichiy, I.A. Tarakanov, R.V. Uskov, "On modeling of the radiation-induced charge effects in the fine dispersed materials of closed-cell structure", Keldysh Institute preprints, 80, 15 pp. (2019)

[11] F.H. Stillinger, B.D. Lubachevsky, "Crystalline-Amorphous Interface Packings for Disks and Spheres”, J. Stat. Phys., 73(3-4), 497-514 (1993).

[12] B.D. Lubachevsky, F.H. Stillinger, "Geometric properties of random disk packings", J. Stat. Phys., 60, 561-583 (1990).

[13] B.D. Lubachevsky, "How to Simulate Billiards and Similar Systems", J. Comput. Phys., 94(2), 255-283 (1991).

[14] M.E. Zhukovskiy, R.V. Uskov, "Hybrid parallelization of the algorithms of radiation cascade transport modeling", Math. Models Comput. Simul., 7, 601-610 (2015). https://doi.org/10.1134/S2070048215060101

[15] V.A. Egorova, F.N. Voronin, M.E. Zhukovskiy, et al., "Model of Radiation-Induced Thermomechanical Effects in Heterogeneous Finely Dispersed Materials", Math. Models Comput. Simul., 12, 729-739 (2020). https://doi.org/10.1134/S2070048220050063.

[16] T. Mitchell, Machine Learning, McGraw-Hill Science/Engineering/Math, (1997).

[17] Simon Haykin, Neural networks. A comprehensive Foundation. Second edition, Prentice hall, Upper Saddle River, New Jersey 07458, (1999).

[18] J. Nocedal, S.J. Wright, Numerical Optimization. 2nd edition. USA: Springer, (2006). ISBN 978-0-387-30303-1. 
[19] A.V. Berezin, N.S. Kellin, M.B. Markov, S.V. Parot'kin, A.V. Sysenko, "The radiative electromagnetic field in the object with the boundary of ideal conductivity", Matem. Mod., 16(3), 3-12 (2004) http://mi.mathnet.ru/eng/mm/v16/i3/p3

[20] A.N. Andrianov, A.V. Berezin, A.S. Vorontsov, K.N. Efimkin, M.B. Markov, "The radiational electromagnetic fields modeling at the multiprocessor computing systems", Matem. Mod., 20(3), 98-114 (2008), http://mi.mathnet.ru/eng/mm/v20/i3/p98

[21] I.P. Bezrodnykh, A.P. Tiutnev, V.T. Semenov, Radiatsionnye effecty v kosmose. Ch. 2. Vozdeistvie kosmicheskoi radiatsii na elektrotekhnicheskie materialy, M.: AO "Corporatsiia "VNIIEM" (2016).

Received September 24, 2021 